\title{
Synthesis of Adaptive Robust Controllers for a Class of Nonlinear Systems with Input Saturations
}

\author{
Kenta Oba, ${ }^{1}$ Hidetoshi Oya, ${ }^{2}$ Tomohiro Kubo, ${ }^{3}$ and Tsuyoshi Matsuki ${ }^{4}$ \\ ${ }^{1}$ Graduate School of Advanced Technology and Science, Tokushima University, 2-1 Minamijosanjima, Tokushima 770-8506, Japan \\ ${ }^{2}$ Department of Computer Science, Tokyo City University, 1-28-1 Tamazutsumi, Setagaya, Tokyo 158-8557, Japan \\ ${ }^{3}$ Graduate School of Technology, Industrial and Social Sciences, Tokushima University, 2-1 Minamijosanjima, \\ Tokushima 770-8506, Japan \\ ${ }^{4}$ Department of Electronics and Control Engineering, National Institute of Technology, Niihama College, \\ 7-1 Yagumo, Niihama, Ehime 792-8580, Japan \\ Correspondence should be addressed to Hidetoshi Oya; hide@tcu.ac.jp
}

Received 4 July 2016; Revised 29 November 2016; Accepted 2 February 2017; Published 4 October 2017

Academic Editor: Mohammad D. Aliyu

Copyright (c) 2017 Kenta Oba et al. This is an open access article distributed under the Creative Commons Attribution License, which permits unrestricted use, distribution, and reproduction in any medium, provided the original work is properly cited.

\begin{abstract}
This paper deals with a design problem of an adaptive robust controller for a class of nonlinear systems with specified input saturations. For the nonlinear system under consideration, the nonlinearity means unknown perturbations and satisfies the matching condition. In this paper, we show that sufficient conditions for the existence of the proposed adaptive robust controller giving consideration to input saturations are given in terms of linear matrix inequalities (LMIs). Finally, simple illustrative examples are shown.
\end{abstract}

\section{Introduction}

It is well known that robust control for uncertain dynamical systems is very important topic for the control engineering community, and therefore various robust control problems have been well studied (see [1] and references therein). Moreover many robust controllers, achieving some robust performance such as mixed $\mathscr{H}^{2} / \mathscr{H}^{\infty}$ control [2] and guaranteed cost control [3], have been suggested. Additionally, Šiljak and Stipanovic [4] and Zuo et al. [5] have presented results of robust stability and stabilization for linear continuoustime and discrete-time system under nonlinear perturbations using the linear matrix inequalities (LMIs). Although almost all of these controllers consist of a state feedback with a fixed feedback gain, the fixed feedback gain can be derived by considering the worst case for unknown parameter variations. In contrast with the above-mentioned control strategies, adaptive robust controllers which are adjusted by parameter updating laws have also been presented (e.g., $[6,7])$. In the work of Maki and Hagino [6], an adaptation mechanism for improving transient behavior has been introduced, and the robust controller includes fixed gain parameters and adjustable ones which are tuned by some updating laws. Moreover, Oya and Hagino [7] have proposed a robust controller with adaptive compensation inputs, and the adaptive compensation input consists of a state feedback with an adaptive gain and a compensation input. One can see that these adaptive robust controllers have time-varying adjustable parameters which are tuned by adjustment laws.

On the other hand in practical systems, there are some constraints such as some limit of actuators and electric saturations for electronic circuits. If the constraint conditions are violated, they cannot generate the desired response, and at worst the system becomes unstable. From these viewpoints, analysis and/or controller design of dynamical systems with constraint conditions are very important issue, and there are a large number of the existing results such as reachable and controllable sets [8], Model Predictive Control (MPC) [9], and saturation-dependent Lyapunov functions [10]. Gilbert and Tan [11] have proposed a general theory which pertains to the maximal output admissible sets $\mathcal{O}_{\infty}$ and $\mathcal{O}_{\infty}^{c}$ for linear systems with state and input constraints. However, so far the 
design problem of adaptive robust controllers with adjustable parameters for uncertain dynamical systems with input saturations has little been considered as far as we know.

In this paper, on the basis of the work of Oya and Hagino [7] we propose a new LMI-based design method of adaptive robust controllers for a class of nonlinear systems with input saturations. For the nonlinear system considered here, the nonlinearity means unknown perturbations and satisfies the well-known matching condition [12]. By using the concept of state reachable sets, the proposed controller can flexibly be adjusted by parameter updating laws [7] and satisfies the specified input saturations. Namely, the result of this paper is a natural extension of the existing result [7]. In this paper, we show that sufficient conditions for the existence of the proposed adaptive robust controller are given in terms of LMIs. Thus, the proposed adaptive robust controller can easily be derived by solving LMIs, and this is also an advantage for the proposed controller design approach.

This paper is organized as follows. In Section 2, notation and useful lemmas which are used in this paper are shown, and in Section 3 we introduce the class of nonlinear systems with input constraints under consideration. Section 4 contains the main results. Finally we show simple illustrative examples to verify the effectiveness of the proposed robust controller.

\section{Preliminaries}

In this section, notations and useful and well-known lemmas (see $[13,14]$ for details) which are used in this paper are shown.

In the paper, the following notations are used: For a matrix $\mathscr{A}$, the inverse of matrix $\mathscr{A}$ and its transpose are denoted by $\mathscr{A}^{-1}$ and $\mathscr{A}^{T}$, respectively. Additionally $H_{e}\{\mathscr{A}\}$ and $I_{n}$ mean $\mathscr{A}+\mathscr{A}^{T}$ and $n$-dimensional identity matrix, respectively, and the notation $\operatorname{diag}\left(\mathscr{A}_{1}, \ldots, \mathscr{A}_{\mathscr{N}}\right)$ represents a block diagonal matrix composed of matrices $\mathscr{A}_{i}$ for $i=$ $1, \ldots, \mathscr{N}$. Trace $\{\mathscr{A}\}$ represents trace of $\mathscr{A}$. For real symmetric matrices $\mathscr{A}$ and $\mathscr{B}, \mathscr{A}>\mathscr{B}$ (resp., $\mathscr{A} \geq \mathscr{B}$ ) means that $\mathscr{A}-\mathscr{B}$ is positive (resp., nonnegative) definite matrix. For a vector $\alpha \in \mathfrak{R}^{n},\|\alpha\|$ denotes standard Euclidian norm and for a matrix $\mathscr{A},\|\mathscr{A}\|$ represents its induced norm. All the eigenvalues of the matrix $\mathscr{A}$ are denoted by $\lambda\{\mathscr{A}\}$, and $\lambda_{\max }\{\mathscr{A}\}$ means the maximum value of the eigenvalues. Additionally, for a symmetric positive definite matrix $\mathscr{P} \in$ $\mathfrak{R}^{n \times n}, \mathscr{E}(\mathscr{P})$ represents a region $\mathscr{E}(\mathscr{P})=\left\{\zeta \in \mathfrak{R}^{n} \mid \zeta^{T} \mathscr{P} \zeta \leq\right.$ $1\}$. The symbols “ڤ” and “ $\star$ " mean equality by definition and symmetric blocks in matrix inequalities, respectively.

Next, we show some useful lemmas which are used in this paper.

Lemma 1. For arbitrary vectors $\lambda$ and $\xi$ and the matrices $\mathscr{G}$ and $\mathscr{H}$ which have appropriate dimensions, the following inequality holds:

$$
2 \lambda^{T} \mathscr{G} \Delta(t) \mathscr{H} \xi \leq 2\left\|\mathscr{G}^{T} \lambda\right\|\|\mathscr{H} \xi\|,
$$

where $\Delta(t)$ with appropriate dimensions is a time-varying unknown matrix satisfying $\|\Delta(t)\| \leq 1.0$.
Lemma 2 ( $\mathcal{S}$-procedure [14]). Let $\mathscr{F}(x), \mathscr{S}(x)$, and $\mathscr{H}(x)$ be three arbitrary quadratic forms over $\mathfrak{R}^{n}$. Then $\mathscr{F}(x)<$ $0 \forall x \in \Re^{n}$ satisfying $\mathscr{G}(x) \leq 0$ and $\mathscr{H}(x) \leq 0$ if there exist nonnegative scalars $\tau_{1}$ and $\tau_{2}$ such that

$$
\mathscr{F}(x)-\tau_{1} \mathscr{G}(x)-\tau_{2} \mathscr{H}(x) \leq 0 \quad \forall x \in \mathfrak{R}^{n} .
$$

Lemma 3 (Schur complement [14]). For a given constant real symmetric matrix $\Theta$, we consider the following inequality conditions:

(i)

$$
\Theta=\left(\begin{array}{ll}
\Theta_{11} & \Theta_{12} \\
\Theta_{12}^{T} & \Theta_{22}
\end{array}\right) \geq 0,
$$

(ii)

$$
\begin{aligned}
& \Theta_{11}>0 \\
& \Theta_{22}-\Theta_{12}^{T} \Theta_{11}^{-1} \Theta_{12} \geq 0,
\end{aligned}
$$

(iii)

$$
\begin{aligned}
& \Theta_{22}>0, \\
& \Theta_{11}-\Theta_{12} \Theta_{22}^{-1} \Theta_{12}^{T} \geq 0 .
\end{aligned}
$$

Then the inequality condition of $(i)$ is equivalent to inequalities of (ii) and (iii).

\section{Problem Formulation}

Let us consider the nonlinear system represented by the following state equation:

$$
\begin{aligned}
\frac{d}{d t} x(t) & =A x(t)+B u(t)+\xi(x, t)+B_{r} r(t), \\
x(0) & =0
\end{aligned}
$$

where $x(t) \in \mathfrak{R}^{n}, u(t) \in \mathfrak{R}^{m}$, and $r(t) \in \mathfrak{R}^{l}$ are the vectors of the state, the control input, and the reference input, respectively. In (6), the reference input $r(t)$ is assumed to belong to the ellipsoidal set $\mathscr{E}\left(\mathscr{X}_{r}\right)$ defined as

$$
\mathscr{E}\left(\mathscr{X}_{r}\right)=\left\{r \in \mathfrak{R}^{l} \mid r^{T} \mathscr{X}_{r} r \leq 1\right\} .
$$

Note that $\mathscr{X}_{r} \in \mathfrak{R}^{l \times l}$ is a given symmetric positive definite matrix. In addition, the vector $\xi(x, t) \in \mathfrak{R}^{n}$ in (6) means nonlinearity and uncertainty in the controlled system, and it satisfies the relation $\xi(x, t)=B g(x, t)$; that is, a well-known matching condition [12] is satisfied. Although the nonlinear function $g(x, t) \in \mathfrak{R}^{m}$ is "unknown," for the known matrix $\mathscr{G} \in \mathfrak{R}^{m \times n}$, it is assumed to satisfy the following inequality condition $[4,5]$ :

$$
\|g(x, t)\| \leq\|\mathscr{G} x(t)\|
$$

In the sequel, we deal with the case of $m=1$ for simplicity, because the results for the case of $m \geq 2$ can easily be obtained 
by the following result (see Remark 5). Therefore, we consider the following constraint for the control input $u(t) \in \mathfrak{R}^{1}$ :

$$
|u(t)| \leq \mu,
$$

where $\mu$ is a known positive constant, and this relation is equivalent to $\|u(t)\| \leq \mu$.

Now for the nonlinear system of (6), we consider the control input $u(t) \in \mathfrak{R}^{m}$ described as

$$
u(t) \triangleq K x(t)+\psi(x, t) .
$$

In (10) $K \in \mathfrak{R}^{m \times n}$ and $\psi(x, t) \in \mathfrak{R}^{m}$ are a fixed feedback gain and an adaptive compensation input, respectively. From (6) and (10), the closed-loop system can be described as

$$
\frac{d}{d t} x(t)=A_{K} x(t)+B \psi(x, t)+B g(x, t)+B_{r} r(t),
$$

where $A_{K} \in \Re^{n \times n}$ is a matrix given by $A_{K}=A+B K$.

Next we consider a state reachable set $\mathscr{R}(x, t)$ for the nonlinear system of (6). By using a symmetric positive definite matrix $\mathscr{P} \in \mathfrak{R}^{n \times n}$, we introduce the quadratic function $\mathscr{V}(x, t) \triangleq x^{T}(t) \mathscr{P} x(t)$ and consider the time derivative of the quadratic function $\mathscr{V}(x, t)$ along the trajectory of the closedloop system of (11). The ellipsoid $\mathscr{E}(\mathscr{P})$ contains the state reachable set $\mathscr{R}(x)$; that is, $\mathscr{R}(x) \subset \mathscr{E}(\mathscr{P})$ provided that, for any $x(t)$ and $r(t)$ satisfying $\mathscr{V}(x, t) \geq 1$ and $r^{T}(t) \mathscr{X}_{r} r(t) \leq 1$, the following inequality condition holds [14]:

$$
\frac{d}{d t} \mathscr{V}(x, t) \leq 0 .
$$

Therefore, our objective in this paper is to develop an LMI-based design procedure of the proposed adaptive robust controller which guarantees the internal stability of the resultant closed-loop system of (11) and the input constraint of (9). In this paper, by using the concept of the state reachable set, we derive an LMI-based design method for the fixed gain $K \in \mathfrak{R}^{m \times n}$ and the adaptive compensation input $\psi(x, t) \in$ $\mathfrak{R}^{m}$ such that the closed-loop system of (11) achieves not only internal stability but also the control input constraint of $\|u(t)\| \leq \mu$.

\section{Synthesis of Adaptive Robust Controllers}

In this section, we show an LMI-based design method of the fixed feedback gain $K \in \Re^{m \times n}$ and the adaptive compensation input $\psi(x, t) \in \mathfrak{R}^{m}$ which ensure internal stability of the closed-loop system of (11) and satisfy the input constraint $\|u(t)\| \leq \mu$.

4.1. Analysis of State Reachable Set $\mathscr{R}(x)$. The time derivative of the quadratic function $\mathscr{V}(x, t)$ can be expressed as

$$
\begin{aligned}
\frac{d}{d t} \mathscr{V}(x, t)= & x^{T}(t)\left(A_{K}^{T} \mathscr{P}+\mathscr{P} A_{K}\right) x(t) \\
& +2 x^{T}(t) \mathscr{P} B \psi(x, t) \\
& +2 x^{T}(t) \mathscr{P} B g(x, t) \\
& +2 x^{T}(t) \mathscr{P} B_{r} r(t) .
\end{aligned}
$$

Firstly, we consider the case of $B^{T} \mathscr{P} x(t) \neq 0$. Then by defining the adaptive compensation input $\psi(x, t)$ of $(10)$ as

$$
\psi(x, t) \triangleq-\frac{\|\mathscr{G} x(t)\|}{\left\|B^{T} \mathscr{P} x(t)\right\|} B^{T} \mathscr{P} x(t)
$$

and using Lemma 1, the inequality

$$
\begin{aligned}
\frac{d}{d x} \mathscr{V}(x, t) \\
\leq x^{T}(t)\left[H_{e}\left\{\mathscr{P} A_{K}\right\}\right] x(t) \\
\quad+2 x^{T}(t) \mathscr{P} B\left(-\frac{\|\mathscr{G} x(t)\|}{\left\|B^{T} \mathscr{P} x(t)\right\|} B^{T} \mathscr{P} x(t)\right) \\
\quad+2\left\|B^{T} \mathscr{P} x(t)\right\|\|\mathscr{G} x(t)\|+2 x^{T}(t) \mathscr{P} B_{r} r(t) \\
=x^{T}(t)\left[H_{e}\left\{\mathscr{P} A_{K}\right\}\right] x(t)+2 x^{T}(t) \mathscr{P} B_{r} r(t)
\end{aligned}
$$

is derived. Moreover, one can see that the inequality of (15) can be represented as

$$
\begin{aligned}
\frac{d}{d x} \mathscr{V}(x, t) & \leq\left(\begin{array}{c}
x(t) \\
r(t)
\end{array}\right)^{T}\left(\begin{array}{cc}
H_{e}\left\{\mathscr{P} A_{K}\right\} & \mathscr{P} B_{r} \\
\star & 0
\end{array}\right)\left(\begin{array}{c}
x(t) \\
r(t)
\end{array}\right) \\
& =\left(\begin{array}{c}
x(t) \\
r(t)
\end{array}\right)^{T} \Psi(K, \mathscr{P})\left(\begin{array}{c}
x(t) \\
r(t)
\end{array}\right),
\end{aligned}
$$

where $\Psi(K, \mathscr{P}) \in \mathfrak{R}^{(n+l) \times(n+l)}$ is given by

$$
\Psi(K, \mathscr{P}) \triangleq\left(\begin{array}{cc}
A_{K}^{T} \mathscr{P}+\mathscr{P} A_{K} & \mathscr{P} B_{r} \\
\star & 0
\end{array}\right) .
$$

On the other hand, if $B^{T} \mathscr{P} x(t)=0$ is satisfied, then we see from (13) that the following equation holds:

$$
\begin{aligned}
\frac{d}{d t} \mathscr{V}(x, t)= & x^{T}(t)\left(A_{K}^{T} \mathscr{P}+\mathscr{P} A_{K}\right) x(t) \\
& +2 x^{T}(t) \mathscr{P} B_{r} r(t) .
\end{aligned}
$$

Note that, in this case, the adaptive compensation input $\psi(x, t) \in \mathbb{R}^{m}$ can be defined as

$$
\psi(x, t) \triangleq \psi\left(x, t_{\delta}\right)
$$

where $t_{\delta}$ is given by $t_{\delta}=\lim _{\delta \rightarrow 0}(t-\delta)$ [6]. Note that the norm of $\psi(x, t)$ can be expressed as $\|\psi(x, t)\|=\|\mathscr{G} x(t)\|$.

From the above discussion, in order for the state reachable set $\mathscr{R}(x)$ of the system of $(6)$ to belong to $\mathscr{E}(\mathscr{P})$, the relation

$$
\left(\begin{array}{l}
x(t) \\
r(t)
\end{array}\right)^{T} \Psi(K, \mathscr{P})\left(\begin{array}{l}
x(t) \\
r(t)
\end{array}\right) \leq 0
$$


should be satisfied. Therefore we introduce $\mathscr{F}_{k}(x, r)(k=$ $0,1,2)$ defined as

$$
\begin{aligned}
& \mathscr{F}_{0}(x, r) \triangleq\left(\begin{array}{l}
x(t) \\
r(t)
\end{array}\right)^{T} \Psi(K, \mathscr{P})\left(\begin{array}{l}
x(t) \\
r(t)
\end{array}\right), \\
& \mathscr{F}_{1}(x, r) \triangleq\left(\begin{array}{l}
x(t) \\
r(t)
\end{array}\right)^{T}\left(\begin{array}{rr}
-\mathscr{P} & 0 \\
0 & 0
\end{array}\right)\left(\begin{array}{l}
x(t) \\
r(t)
\end{array}\right)+1, \\
& \mathscr{F}_{2}(x, r) \triangleq\left(\begin{array}{l}
x(t) \\
r(t)
\end{array}\right)^{T}\left(\begin{array}{ll}
0 & 0 \\
0 & X_{r}
\end{array}\right)\left(\begin{array}{l}
x(t) \\
r(t)
\end{array}\right)-1 .
\end{aligned}
$$

Note that the inequality condition of $(20), \mathscr{V}(x, t) \geq 1.0$, and $r(t) \subset \mathscr{E}\left(\mathscr{X}_{r}\right)$ correspond to $\mathscr{F}_{k}(x, r) \leq 0(k=0,1,2)$, respectively. Therefore we consider

$$
\begin{aligned}
& \mathscr{F}_{0}(x, r) \leq 0 \\
& \text { subject to } \mathscr{F}_{1}(x, r) \leq 0, \\
& \mathscr{F}_{2}(x, r) \leq 0 .
\end{aligned}
$$

By using Lemma 2 ( $\mathcal{S}$-procedure), the condition of (22) is equivalent to

$$
\begin{aligned}
& \left(\begin{array}{c}
x(t) \\
r(t)
\end{array}\right)^{T}\left(\begin{array}{cc}
H_{e}\left\{\mathscr{P} A_{K}\right\}+\tau_{1} \mathscr{P} & \mathscr{P} B_{r} \\
\star & -\tau_{2} \mathscr{X}_{r}
\end{array}\right)\left(\begin{array}{l}
x(t) \\
r(t)
\end{array}\right) \\
& \quad-\tau_{1}+\tau_{2} \leq 0 .
\end{aligned}
$$

Namely, if there exist the positive definite symmetric matrix $\mathscr{P} \in \mathfrak{R}^{n \times n}$, the fixed gain matrix $K \in \mathfrak{R}^{m \times n}$, and the positive scalars $\tau_{1}$ and $\tau_{2}$ satisfying

$$
\left(\begin{array}{ccc}
H_{e}\left\{\mathscr{P} A_{K}\right\}+\tau_{1} \mathscr{P} & \mathscr{P} B_{r} & 0 \\
\star & -\tau_{2} \mathscr{X}_{r} & 0 \\
\star & \star & -\tau_{1}+\tau_{2}
\end{array}\right) \leq 0,
$$

then $\mathscr{R}(x) \subset \mathscr{E}(\mathscr{P})$. Clearly we must have $\tau_{1} \geq \tau_{2}$. If the inequality condition of (24) is satisfied for some $\left(\tau_{1}^{\prime}, \tau_{2}^{\prime}\right)$, then it holds for all $\tau_{1}^{\prime} \geq \tau_{2} \geq \tau_{2}^{\prime}$. Therefore, we can assume without loss of generality that $\tau_{1}=\tau_{2}=\tau$, and the condition of (24) can be rewritten as the following form [14]:

$$
\left(\begin{array}{cc}
H_{e}\left\{\mathscr{P} A_{K}\right\}+\tau \mathscr{P} & \mathscr{P} B_{r} \\
\star & -\tau \mathscr{X}_{r}
\end{array}\right) \leq 0 .
$$

Here by introducing the complementary matrices $\mathcal{S} \triangleq$ $\mathscr{P}^{-1}$ and $\mathscr{W} \triangleq K \mathcal{S}$ and pre- and postmultiplying (25) by $\operatorname{diag}\left(\mathcal{S}, I_{m}\right)$, we have

$$
\left(\begin{array}{cc}
H_{e}\{A \mathcal{S}+B \mathscr{W}\}+\tau \mathcal{S} & B_{r} \\
\star & -\tau \mathscr{X}_{r}
\end{array}\right) \leq 0 .
$$

Consequently, if there exist the symmetric positive definite matrix $\mathcal{S} \in \mathfrak{R}^{n \times n}$, the matrix $\mathscr{W} \in \mathfrak{R}^{m \times n}$, and the positive scalar $\tau$ which satisfy the matrix inequality of (26), then the state reachable set $\mathscr{R}(x)$ for the closed-loop system of (11) is included in $\mathscr{E}(\mathscr{P})$.
4.2. Analysis of Input Constraints. In Section 4.1, the condition for achieving the relation $\mathscr{R}(x) \subset \mathscr{E}(\mathscr{P})$ has been derived. Next we consider the input constraints.

From (10) and (14), we find that the control input $u(t) \in$ $\mathfrak{R}^{1}$ can be written as

$$
u(t)=K x(t)-\frac{\|\mathscr{G} x(t)\|}{\left\|B^{T} \mathscr{P} x(t)\right\|} B^{T} \mathscr{P} x(t) .
$$

Moreover, one can see from Section 4.1 that the state reachable set $\mathscr{R}(x)$ belongs to $\mathscr{E}(\mathscr{P})$.

Firstly, we consider the 1st term of the right hand side of (27). Taking the Euclidian norm of $K x(t)$, we obtain

$$
\begin{aligned}
\max _{t}\|K x(t)\| & =\max _{t}\left\|\mathscr{W} \mathcal{S}^{-1} x(t)\right\| \\
& \leq \max _{\zeta \in \mathscr{C}(\mathscr{P})}\left\|\mathscr{W} \mathcal{S}^{-1} \zeta\right\| \\
& =\max _{\zeta \in \mathscr{E}(\mathscr{P})}\left\|\mathscr{W} \mathcal{S}^{-1 / 2} \mathcal{S}^{-1 / 2} \zeta\right\| \\
& \leq \sqrt{\lambda_{\max }\left\{\mathcal{S}^{-1 / 2} \mathscr{W}^{T} \mathscr{W} \mathcal{S}^{-1 / 2}\right\} .}
\end{aligned}
$$

Furthermore, one can easily see that, for the 2 nd term of the right hand side of (27), the inequality

$$
\max _{t}\left\|\frac{\|\mathscr{G} x(t)\|}{\left\|B^{T} \mathscr{P} x(t)\right\|} B^{T} \mathscr{P} x(t)\right\|=\max _{t}\|\mathscr{G} x(t)\|
$$

is satisfied. Additionally, by using the similar way to the calculation of (28), we have

$$
\begin{aligned}
\max _{t}\|\mathscr{G} x(t)\| & \leq \max _{\zeta \in \mathscr{C}(\mathscr{P})}\|\mathscr{G} \zeta\|=\max _{\zeta \in \mathscr{E}(\mathscr{P})}\left\|\mathscr{G} \mathcal{S}^{1 / 2} \mathcal{S}^{-1 / 2} \zeta\right\| \\
& \leq \sqrt{\lambda_{\max }\left\{\mathcal{S}^{1 / 2} \mathscr{S}^{T} \mathscr{G} \mathcal{S}^{1 / 2}\right\}} .
\end{aligned}
$$

From (28) and (30), in order to satisfy the specified constraint $\|u(t)\| \leq \mu$, the following inequalities should be satisfied:

$$
\begin{array}{r}
\sqrt{\lambda_{\max }\left\{\mathcal{S}^{\left.-1 / 2 \mathscr{W}^{T} \mathscr{W} \mathcal{S}^{-1 / 2}\right\}}\right.} \leq \mu_{K}, \\
\sqrt{\lambda_{\max }\left\{\mathcal{S}^{1 / 2} \mathscr{G}^{T} \mathscr{G} \mathcal{S}^{1 / 2}\right\}} \leq \mu_{L}, \\
\mu_{K}+\mu_{L} \leq \mu .
\end{array}
$$

In this paper, in order to derive an LMI-based design method, we introduce the conditions

$$
\begin{gathered}
\mathcal{S}^{-1 / 2} \mathscr{W}^{T} \mathscr{W} \mathcal{S}^{-1 / 2} \leq \mu_{K}^{2} I_{n}, \\
\mathcal{S}^{1 / 2} \mathscr{G}^{T} \mathscr{G} \mathcal{S}^{1 / 2} \leq \mu_{L}^{2} I_{n}, \\
\mu_{K}^{2}+\mu_{L}^{2} \leq \mu,
\end{gathered}
$$

instead of the inequalities of (31). Note that the 1 st and the 2 nd inequalities of (32) are equivalent to them of (31), and the $3 \mathrm{rd}$ inequality of (32) is a sufficient condition of the $3 \mathrm{rd}$ one of (31). Thus, if the conditions of (32) hold, then the inequalities 
of (31) are also satisfied. Namely, if there exist the positive scalars $\mu_{K}$ and $\mu_{L}$ satisfying the inequalities of (32), then the input constraint is also satisfied.

Now, by introducing the complementary variables $\mu_{K}^{\star}$ and $\mu_{L}^{\star}$ we consider the following inequalities:

$$
\begin{aligned}
\mathcal{S}^{-1 / 2} \mathscr{W}^{T} \mathscr{W} \mathcal{S}^{-1 / 2} & \leq \mu_{K}^{\star} I_{n}, \\
\mathcal{S}^{1 / 2} \mathscr{G}^{T} \mathscr{G} \mathcal{S}^{1 / 2} & \leq \mu_{L}^{\star} I_{n}, \\
\mu_{K}^{\star}+\mu_{L}^{\star} & \leq \mu, \\
\mu_{K}^{\star} & \geq \mu_{K}^{2}, \\
\mu_{L}^{\star} & \geq \mu_{L}^{2} .
\end{aligned}
$$

One can see from Lemma 3 (Schur complement) that the 4 th and 5th inequalities in (33) are equivalent to

$$
\begin{aligned}
& \left(\begin{array}{cc}
-\mu_{K}^{\star} & -\mu_{K} \\
\star & -1
\end{array}\right) \leq 0, \\
& \left(\begin{array}{cc}
-\mu_{L}^{\star} & -\mu_{L} \\
\star & -1
\end{array}\right) \leq 0 .
\end{aligned}
$$

Moreover, by applying Lemma 3 (Schur complement) to the 1st inequality of (33), we obtain

$$
\begin{gathered}
-\mu_{K}^{\star} I_{n}+\mathcal{S}^{-1 / 2} \mathscr{W}^{T} \mathscr{W} \mathcal{S}^{-1 / 2} \leq 0 \Longleftrightarrow \\
-\mu_{K}^{\star} \mathcal{S}+\mathscr{W}^{T} \mathscr{W} \leq 0 \Longleftrightarrow \\
-\mathcal{S}+\frac{1}{\mu_{K}^{\star}} \mathscr{W}^{T} \mathscr{W} \leq 0 \Longleftrightarrow \\
\left(\begin{array}{cc}
-\mathcal{S} & \mathscr{W}^{T} \\
\mathscr{W} & -\mu_{K}^{\star} I_{m}
\end{array}\right) \leq 0 .
\end{gathered}
$$

Additionally, since the matrix $\mathscr{G} \in \mathfrak{R}^{m \times n}$ is known, one can easily calculate the scalar $\gamma_{\mathscr{G}}$ satisfying $\mathscr{G}^{T} \mathscr{G} \leq \gamma_{\mathscr{G}} I_{n}$. Thus, we introduce the inequality condition

$$
-\mu_{L}^{\star} I_{n}+\gamma_{\mathscr{G}} \mathcal{S} \leq 0
$$

and this inequality is a sufficient condition of the 2nd inequality of (33).

From the above discussion, one can see that, for given positive constants $\tau$ and $\gamma_{\mathscr{G}}$, if the symmetric positive definite matrix $\mathcal{S} \in \mathfrak{R}^{n \times n}$, the matrix $\mathscr{W} \in \mathfrak{R}^{m \times n}$, and the positive scalars $\mu_{K}, \mu_{L}, \mu_{K}^{\star}$, and $\mu_{L}^{\star}$ which satisfy LMIs of (26), (34), (35), and (36) are obtained, then the input constraints are satisfied.

Consequently, our main result is summarized as the following theorem.

Theorem 4. Consider the nonlinear system of (6) and the control input of (10).

For given positive constants $\tau$ and $\gamma_{\mathscr{G}}$, if there exist the symmetric positive definite matrix $\mathcal{S} \in \mathfrak{R}^{n \times n}$, the matrix
$\mathscr{W} \in \mathfrak{R}^{m \times n}$, and the positive scalars $\mu_{K}, \mu_{L}, \mu_{K}^{\star}$, and $\mu_{L}^{\star}$ which satisfy the LMIs

$$
\begin{array}{r}
\left(\begin{array}{cc}
H_{e}\{A \mathcal{S}+B \mathscr{W}\}+\tau \mathcal{S} & B_{r} \\
\star & \\
& -\tau \mathscr{X}_{r}
\end{array}\right) \leq 0, \\
\left(\begin{array}{cc}
-\mathcal{S} & \mathscr{W}^{T} \\
\star & -\mu_{K}^{\star} I_{m}
\end{array}\right) \leq 0, \\
\left(\begin{array}{cc}
-\mu_{K}^{\star} & -\mu_{K} \\
\star & -1
\end{array}\right) \leq 0, \\
\left(\begin{array}{cc}
-\mu_{L}^{\star} & -\mu_{L} \\
\star & -1
\end{array}\right) \leq 0, \\
-\mu_{L}^{\star} I_{n}+\gamma_{\mathscr{G}} \mathcal{S} \leq 0, \\
\mu_{K}^{\star}+\mu_{L}^{\star} \leq \mu,
\end{array}
$$

the fixed gain matrix $K \in \mathfrak{R}^{m \times n}$ and the adaptive compensation input $\psi(x, t) \in \mathfrak{R}^{m}$ are determined as

$$
\begin{aligned}
& K=\mathscr{W} \mathcal{S}^{-1}, \\
& \psi(x, t) \\
& \quad= \begin{cases}-\frac{\|\mathscr{G} x(t)\|}{\left\|B^{T} \mathscr{P} x(t)\right\|} B^{T} \mathscr{P} x(t) & \text { if } B^{T} \mathscr{P} x(t) \neq 0 \\
\psi\left(x, t_{\delta}\right) & \text { if } B^{T} \mathscr{P} x(t)=0,\end{cases}
\end{aligned}
$$

where $t_{\delta}=\lim _{\delta>0, \delta \rightarrow 0}(t-\delta)[6]$. Then the closed-loop system of (11) is internally stable and the state reachable set $\mathscr{R}(x)$ belongs to $\mathscr{E}(\mathscr{P})$. Moreover the input constraints for the control input $u(t)$ are also guaranteed.

Remark 5. In this paper, we introduce the assumption $m=1$ and consider the input constraint of (9).

By the way, for the control input $u(t)=\left(u_{1}(t), \ldots, u_{m}(t)\right)^{T}$ there exist the componentwise input constraints; that is,

$$
\left|u_{i}(t)\right| \leq \mu_{i} \quad(i=1, \ldots, m)
$$

where $\mu_{i}$ is a given positive constant. If the positive constant $\mu_{i}$ for the constraints of (39) satisfies $\mu_{i}=1.0$, one can adopt the inequality [14]

$$
\begin{aligned}
u^{T}(t) \mathscr{Z} u(t) & \leq 1, \\
\text { Trace }\{\mathscr{Z}\} & =1,
\end{aligned}
$$

and the proposed design approach can be easily extended to this case.

On the other hand, for the case of $\mu_{i} \neq 1.0$, although we can consider the condition

$$
\begin{aligned}
u^{T}(t) \mathscr{R} u(t) & \leq 1, \\
\mathscr{R}^{1 / 2} & =\operatorname{diag}\left(\mu^{+}, \ldots, \mu^{+}\right),
\end{aligned}
$$

instead of (40), where $\mu^{+}$means $\max _{j}\left\{\mu_{j}\right\}$, then the resulting controller which is obtained by adopting the condition of (41) 
becomes very conservative. Thus the constraint condition of (41) is not desirable, and efficient and tractable conditions corresponding to the constraint of (39) have not been developed. Namely, the design problem for such constraints is one of our future research subjects.

Remark 6. In this paper, we deal with a stabilization problem of an adaptive robust controller for a class of nonlinear systems with input constraints. On the other hand, in the work of Oya and Hagino [7], by introducing an error signal between the time response and the desired one, the robust controller in [7] can achieve not only robust stability but also satisfactory transient behavior. Note that the proposed controller design approach can easily be extended to such control strategy.

\section{Numerical Examples}

In order to demonstrate the efficiency of the proposed robust controller, we have run a simple numerical example.

Let us consider the nonlinear system

$$
\begin{aligned}
\frac{d}{d t} x(t)= & \left(\begin{array}{cc}
-1.0 & 4.0 \\
0.0 & -5.0
\end{array}\right) x(t)+\left(\begin{array}{l}
0.0 \\
2.0
\end{array}\right) u(t) \\
& +\left(\begin{array}{l}
0.0 \\
2.0
\end{array}\right) g(x, t)+\left(\begin{array}{l}
1.0 \\
2.0
\end{array}\right) r(t),
\end{aligned}
$$

with the input constraint

$$
\|u(t)\| \leq 1.0
$$

Additionally in this simulation, the reference input $r(t) \in \mathfrak{R}^{1}$ and $\mathscr{G} \in \mathfrak{R}^{1 \times 2}$ selected as $r(t)=\mathscr{X}_{r}^{-1} \times \cos (\pi t)\left(\mathscr{X}_{r}=2.2\right)$ and $\mathscr{G}=\left(\begin{array}{ll}2.0 & 1.0\end{array}\right)$, respectively; that is, $\gamma_{\mathscr{G}}=4.0$.

Firstly, in order to derive the proposed adaptive robust controller, we select the design parameter $\tau$ in (37) such as $\tau=$ 2.0. Then by solving the LMIs of (37), the symmetric positive definite matrix $\mathcal{S} \in \mathfrak{R}^{2 \times 2}$, the matrix $\mathscr{W} \in \mathfrak{R}^{1 \times 2}$, and the positive constants $\mu_{K}, \mu_{L}, \mu_{K}^{\star}$, and $\mu_{L}^{\star}$ can be calculated as

$$
\begin{aligned}
& \mathcal{S}=\left(\begin{array}{cc}
2.4439 \times 10^{-1} & -2.8670 \times 10^{-2} \\
\star & 8.2520 \times 10^{-2}
\end{array}\right), \\
& \mathscr{W}=\left(-4.4815 \times 10^{-1}-6.3870 \times 10^{-2}\right), \\
& \mu_{K}=9.9729 \times 10^{-1} \text {, } \\
& \mu_{L}=3.8150 \times 10^{-2} \text {, } \\
& \mu_{K}^{\star}=9.9724 \times 10^{-1} \text {, } \\
& \mu_{L}^{\star}=1.8800 \times 10^{-3} .
\end{aligned}
$$

Namely, the following inequality holds:

$$
\mu_{K}^{\star}+\mu_{L}^{\star}=9.9912 \times 10^{-1}\left(=\mu^{\star}\right)<1.0 .
$$

Thus the constraint of (43) for the control input is satisfied, because $\mu^{\star}<\mu$.
By using the symmetric positive definite matrix $\delta \in \mathfrak{R}^{2 \times 2}$ and the matrix $\mathscr{W} \in \mathfrak{R}^{1 \times 2}$ we have

$$
K=\left(\begin{array}{ll}
-2.0072 & -1.4714
\end{array}\right)
$$

Moreover, the symmetric positive definite matrix $\mathscr{P} \in \mathfrak{R}^{2 \times 2}$ can be obtained as

$$
\mathscr{P}=\left(\begin{array}{cc}
4.2676 & 1.4828 \\
\star & 1.2633 \times 10^{-1}
\end{array}\right),
$$

and the state reachable set $\mathscr{R}(x)$ is included in the ellipsoidal set $\mathscr{E}(\mathscr{P})$ described as

$$
\begin{gathered}
\mathscr{E}(\mathscr{P})=\left\{x \in \mathfrak{R}^{n} \mid 4.2676 \times 10^{-1} \times x_{1}^{2}(t)+2.9656\right. \\
\left.\quad \times x_{1}(t) \times x_{2}(t)+1.2633 \times 10^{-1} \times x_{2}^{2}(t) \leq 1.0\right\} .
\end{gathered}
$$

In this example, the initial value of the uncertain nonlinear system of (42) is selected as $x(0)=\left(\begin{array}{ll}0.0 & 0.0\end{array}\right)^{T}$. Moreover, in this simulation, we consider the following two cases for the nonlinear function $g(x, t)$ (note that these two cases in this example mean a nonlinear function of Case 1 and a linear function of Case 2 as a special case for the nonlinearity, respectively):

Case 1.

$$
\begin{aligned}
& g(x, t) \\
& \quad=\left(\sqrt{2} \cos \left(10 \pi x_{1}(t)\right),-\sin \left(5 \pi x_{2}(t)\right)\right) x(t) .
\end{aligned}
$$

Case 2.

$$
g(x, t)= \begin{cases}0 \leq t<1, & g(x, t)=(-\sqrt{2},-1) x(t) \\ 1 \leq t<3, & g(x, t)=(-\sqrt{2}, 1) x(t) \\ 3 \leq t<5, & g(x, t)=(\sqrt{2},-1) x(t) \\ 5 \leq t, & g(x, t)=(\sqrt{2}, 1) x(t) .\end{cases}
$$

The simulation result of this numerical example is shown in Figures 1-8. Figures 1-6 represent time histories of the state and the control input, and in these figures "Conventional" and "Proposed" mean the conventional robust control based on the existing result [7] and the proposed robust controller, respectively. Namely, "Conventional" does not take input constraints into account. Moreover, "Upper bound" and "Lower bound" in Figures 5 and 6 represent the constraint for control input, and "blue" line in Figures 7 and 8 means the ellipsoidal set $\mathscr{E}(\mathscr{P})$.

From these figures, the proposed robust controller and the conventional adaptive robust controller based on the existing result [7] stabilize the dynamical system of (42) in spite of unknown nonlinearities. Moreover, from Figure 6, we find that the proposed adaptive robust controller satisfies the control input saturation. Additionally, one can see from Figures 7 and 8 that the state trajectory is contained in the ellipsoidal set $\mathscr{E}(\mathscr{P})$. Namely, the proposed controller can robustly stabilize the uncertain system of (42) and also satisfy the control input saturation. 


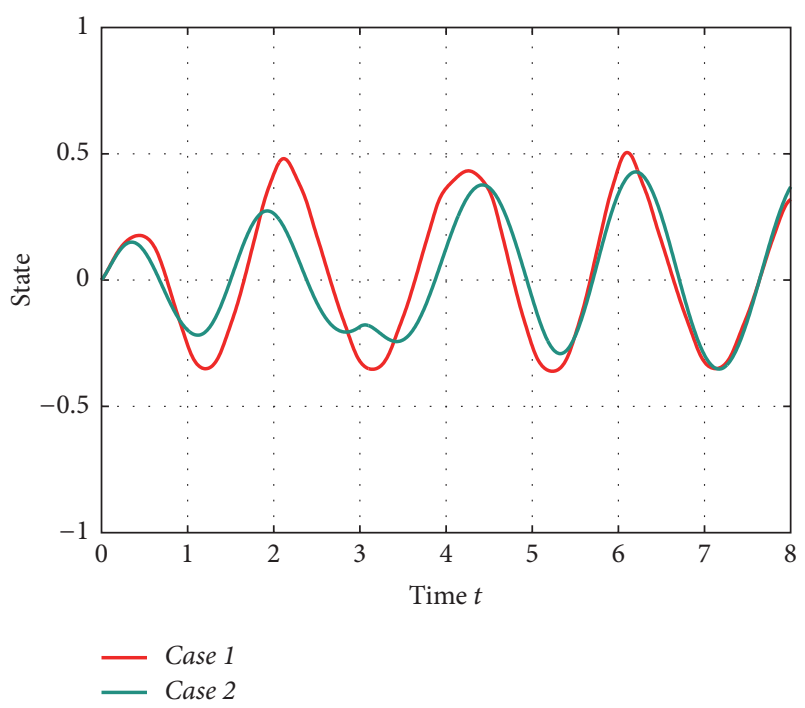

FIgURE 1: Time histories of $x_{1}(t)$ : Conventional.

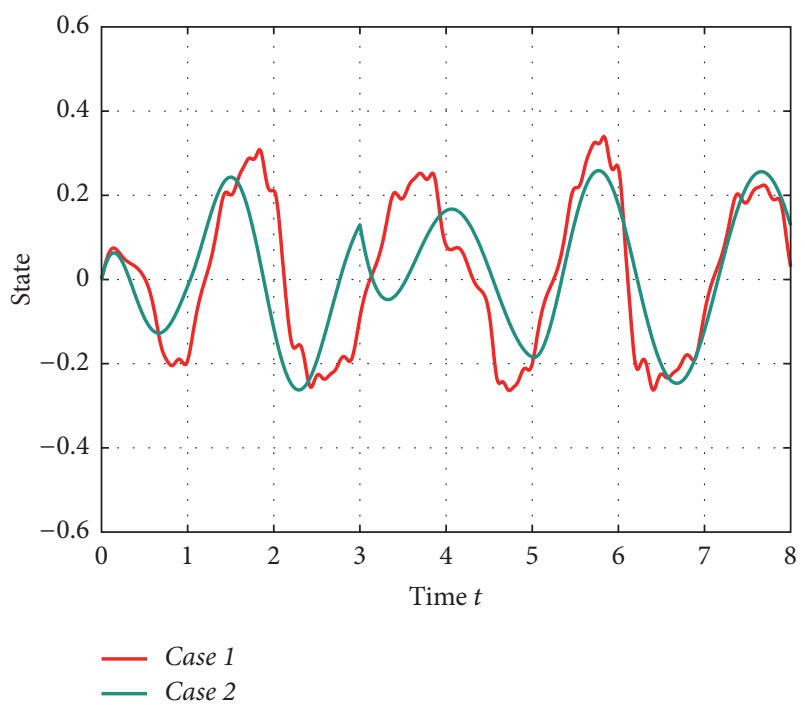

FIgURE 2: Time histories of $x_{2}(t)$ : Conventional.

On the other hand, one can see from Figure 5 that the conventional adaptive robust controller cannot satisfy the input constraints.

Therefore the effectiveness of the proposed adaptive robust controller giving consideration to input constraints for a class of nonlinear systems has been shown.

\section{Conclusion}

In this paper, we have proposed an adaptive robust controller for a class of nonlinear systems with input constraints. The proposed design method is based on LMIs, and thus the proposed adaptive robust controller can easily be obtained by using software such as MATLAB and Scilab. In addition, the effectiveness of the proposed robust controller has been shown by simple numerical examples. Note that the proposed

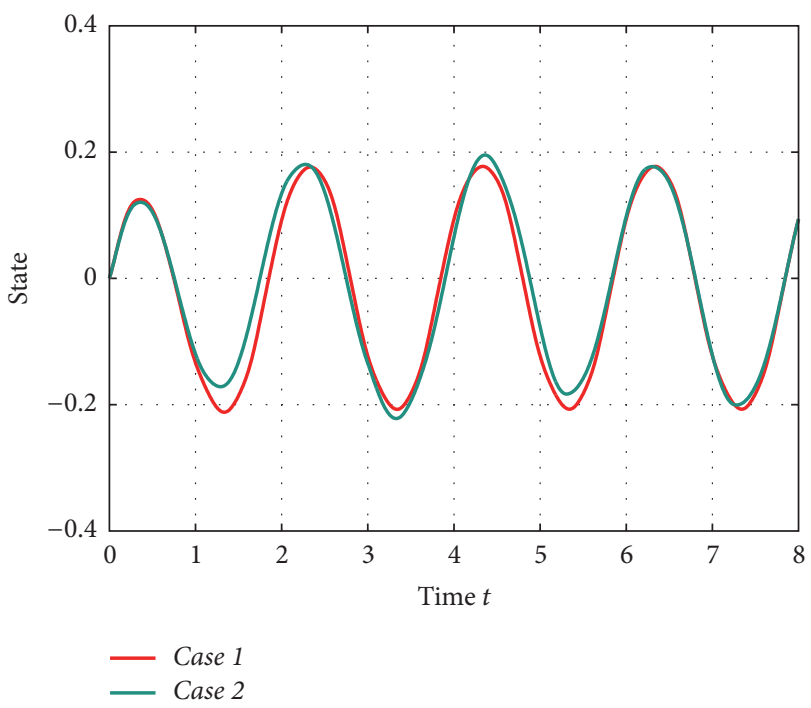

Figure 3: Time histories of $x_{1}(t)$ : Proposed.

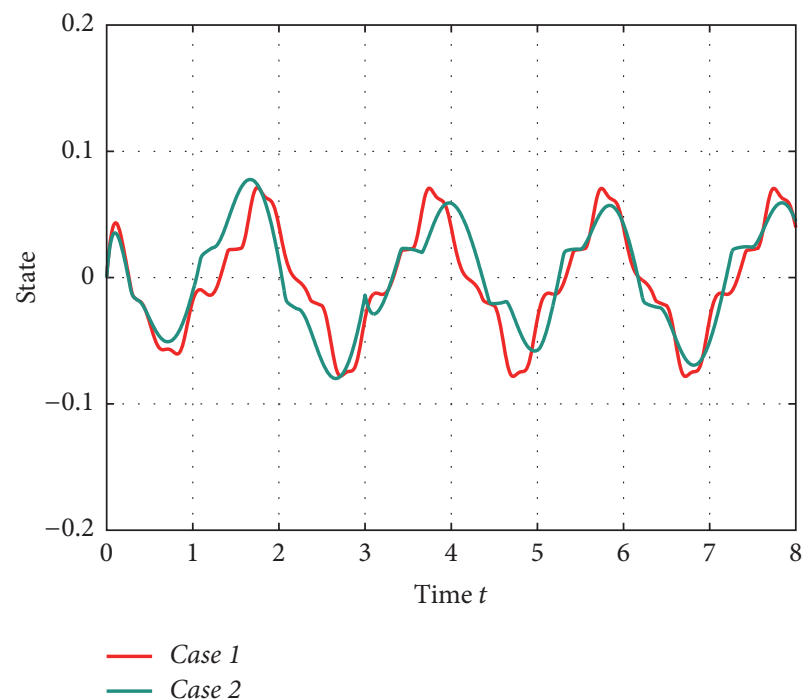

Figure 4: Time histories of $x_{2}(t)$ : Proposed.

adaptive robust controller is an extension of the existing results [7].

In the proposed adaptive robust control strategy, the control input consists of a state feedback with fixed gains and an adaptive compensation input, and the adaptive compensation input is defined as a state feedback with time-varying adjustable parameters.. The advantages of the proposed adaptive robust control scheme are as follows: the proposed robust controller is more flexible and adaptive than conventional fixed gain robust controller, which is designed for the worst case of unknown parameter variations, and satisfies the given input saturations.

In our future work, we will extend the proposed adaptive robust controller synthesis to such a broad class of systems as linear systems with mismatched uncertainties, uncertain nonlinear systems with time delays, and so on. Furthermore, 


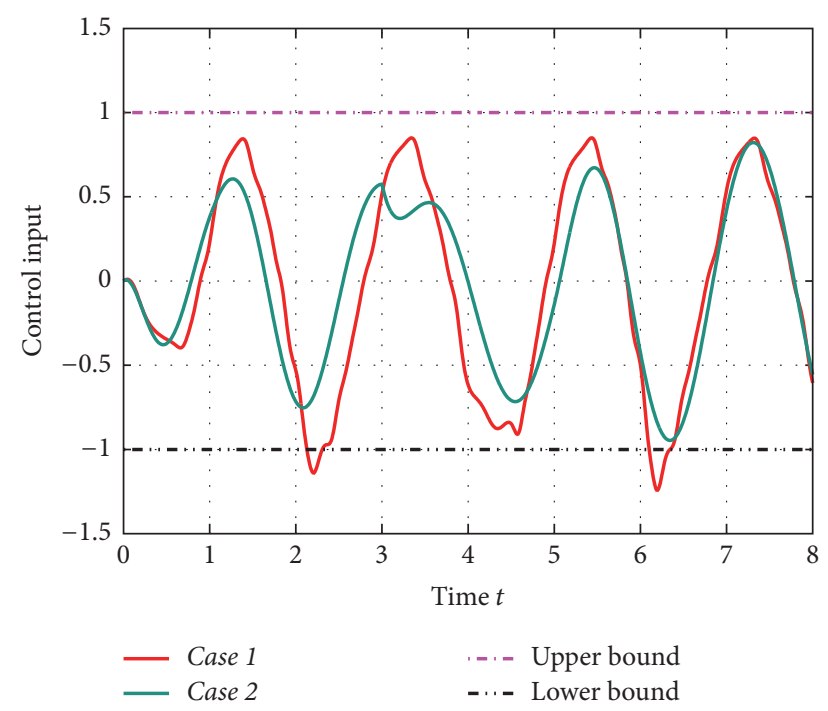

FIgURE 5: Time histories of $u(t)$ : Conventional.

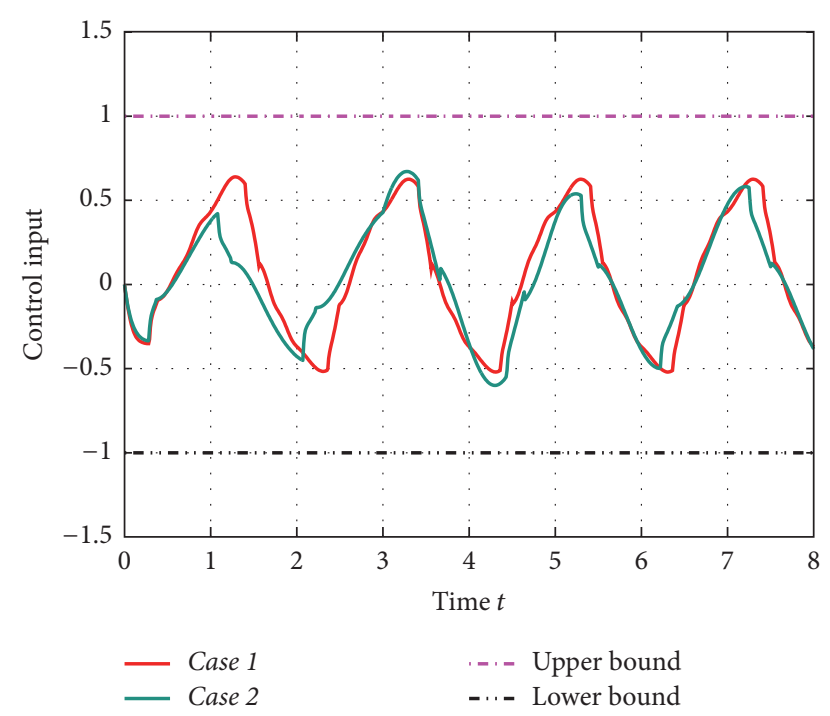

Figure 6: Time histories of $u(t)$ : Proposed.

analysis of the conservativeness of the proposed adaptive robust controller giving consideration to input saturations is also an important issue for our future works. Additionally, the extension of the proposed approach to more general types for constraints such as (39) and the discussion for conservativeness of the proposed design approach are also important future research subjects.

\section{Conflicts of Interest}

The authors declare that there are no conflicts of interest regarding the publication of this paper.

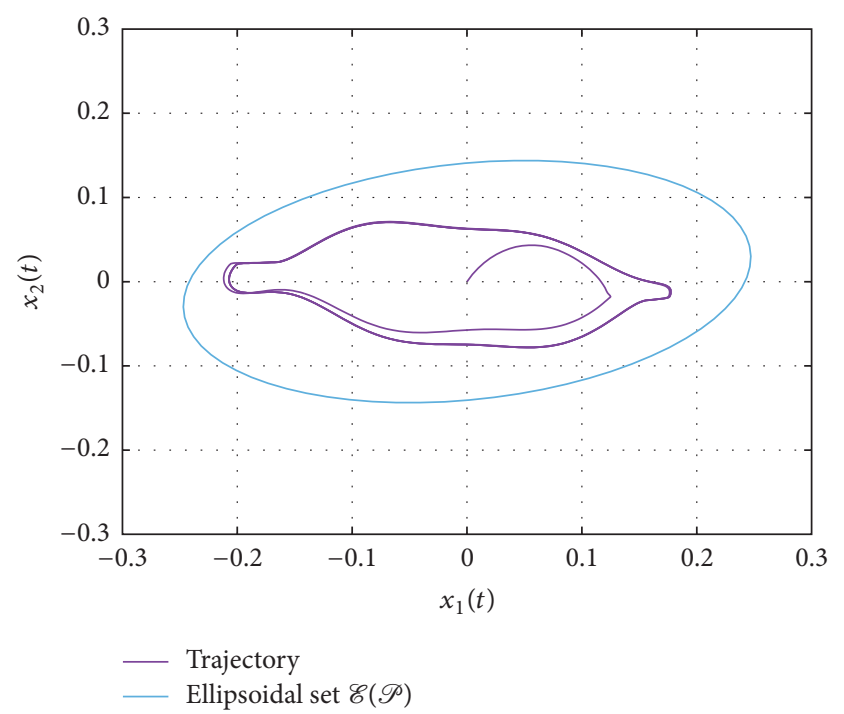

Figure 7: Ellipsoidal set and trajectory of $x(t)$ : Case 1 .

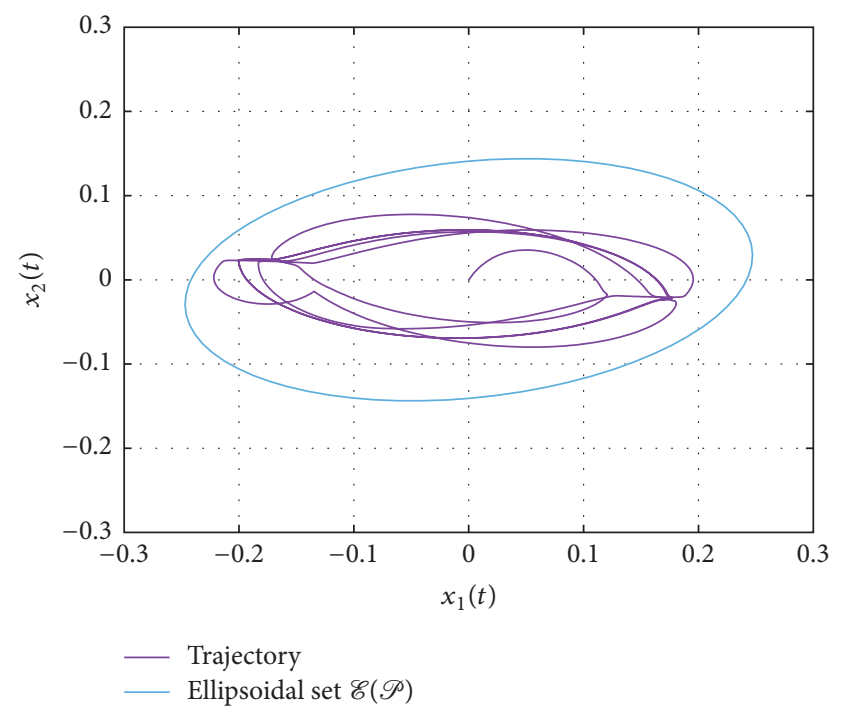

FIGURE 8: Ellipsoidal set and trajectory of $x(t)$ : Case 2.

\section{Acknowledgments}

The authors would like to thank Professor Mohammad D. Aliyu, Associate Editor, with Ecole Polytechnique de Montreal for his valuable and helpful comments that greatly contributed to this paper.

\section{References}

[1] K. Zhou, J. C. Doyle, and K. Glover, Robust and Optimal Control, Prentice Hall, 1996.

[2] P. P. Khargonekar and M. A. Rotea, "Mixed $H_{2} / H_{\infty}$ control, a convex optimization approach," IEEE Transactions on Automatic Control, vol. 36, no. 7, pp. 824-837, 1991.

[3] I. R. Petersen and D. C. McFarlane, "Optimal guaranteed cost control and filtering for uncertain linear systems," IEEE 
Transactions on Automatic Control, vol. 39, no. 9, pp. 1971-1977, 1994.

[4] D. D. Šiljak and D. M. Stipanovic, "Robust stabilization of nonlinear systems: the LMI approach," Mathematical Problems in Engineering, vol. 6, no. 5, pp. 461-493, 2000.

[5] Z. Zuo, J. Wang, and L. Huang, "Robust stabilization for nonlinear discrete-time systems," International Journal of Control, vol. 77, no. 4, pp. 384-388, 2004.

[6] M. Maki and K. Hagino, "Robust control with adaptation mechanism for improving transient behaviour," International Journal of Control, vol. 72, no. 13, pp. 1218-1226, 1999.

[7] H. Oya and K. Hagino, "Robust control with adaptive compensation input for linear uncertain systems," IEICE Transactions on Fundamentals of Electronics, Communications and Computer Sciences, vol. 86, no. 6, pp. 1517-1524, 2003.

[8] J. E. Gayek, "A survey of techniques for approximating reachable and controllable sets", in Proceedings of the 30th IEEE Conference on Decision and Control, pp. 1724-1729, IEEE, Brighton, UK, December 1991.

[9] D. Q. Mayne, J. B. Rawlings, C. V. Rao, and P. O. M. Scokaert, "Constrained model predictive control: stability and optimality," Automatica, vol. 36, no. 6, pp. 789-814, 2000.

[10] Y.-Y. Cao and Z. Lin, "Stability analysis of discrete-time systems with actuator saturation by a saturation-dependent Lyapunov function," Automatica, vol. 39, no. 7, pp. 1235-1241, 2003.

[11] E. G. Gilbert and K. T. Tan, "Linear systems with state and control constraints: the theory and application of maximal output admissible sets," IEEE Transactions on Automatic Control, vol. 36, no. 9, pp. 1008-1020, 1991.

[12] F. Lin, R. D. Brandt, and J. Sun, "Robust control of nonlinear systems: compensating for uncertainty," International Journal of Control, vol. 56, no. 6, pp. 1453-1459, 1992.

[13] F. R. Gantmacher, The Theory of Matrices, vol. 1, Chelsea Publishing, New York, NY, USA, 1960.

[14] S. Boyd, L. El Ghaoui, E. Feron, and V. Balakrishnan, Linear Matrix Inequalities in System and Control Theory, SIAM Studies in Applied Mathematics, 1994. 


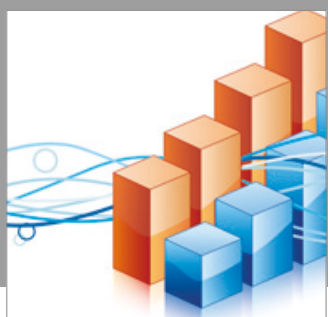

Advances in

Operations Research

vatersals

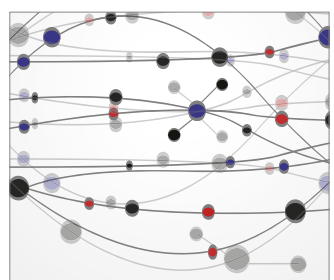

\section{The Scientific} World Journal
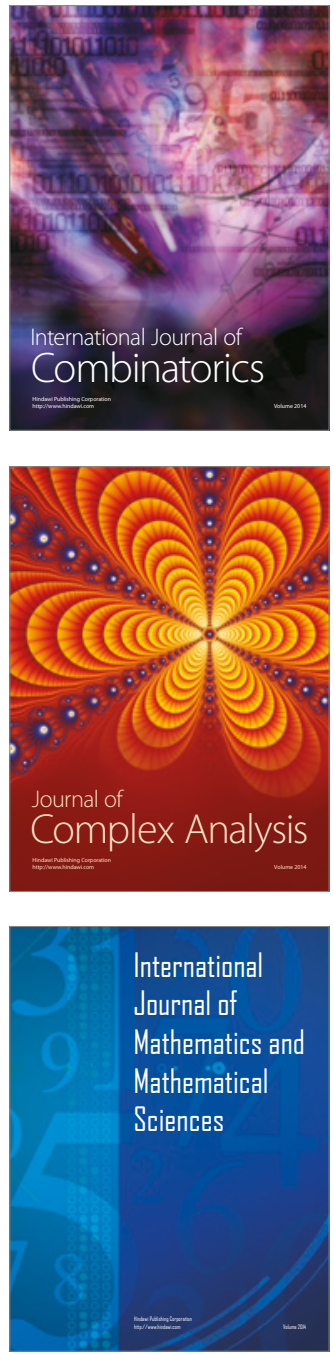
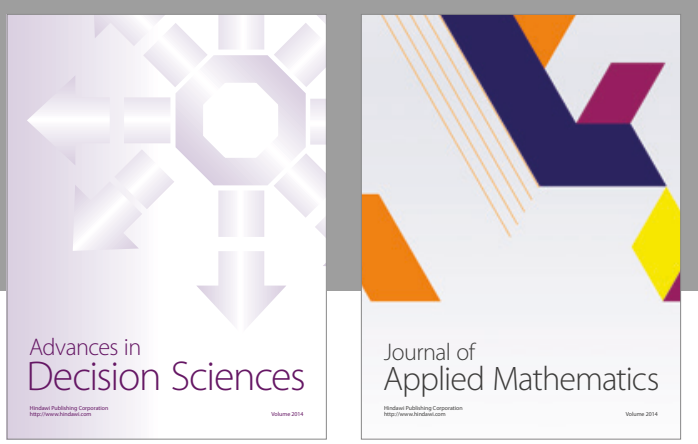

Algebra

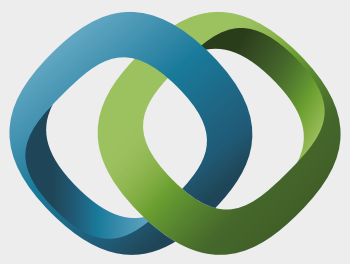

\section{Hindawi}

Submit your manuscripts at

https://www.hindawi.com
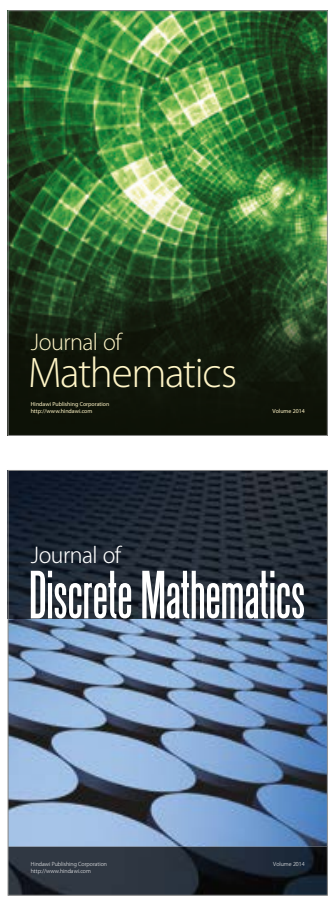

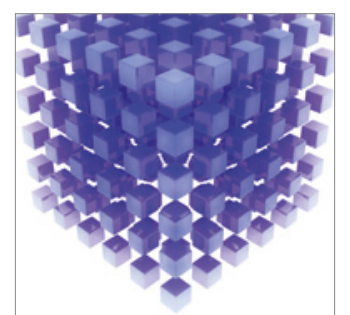

Mathematical Problems in Engineering
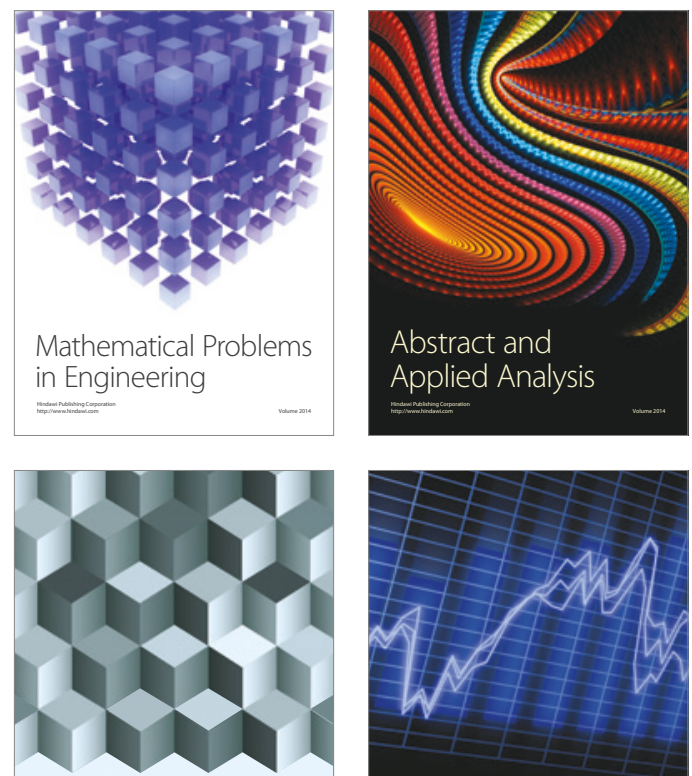

Journal of

Function Spaces

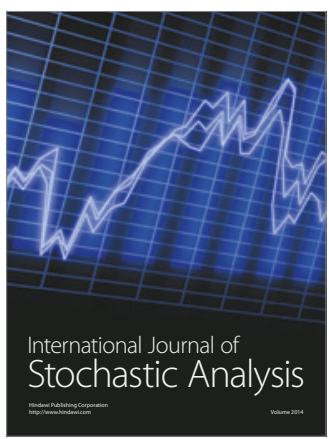

Probability and Statistics
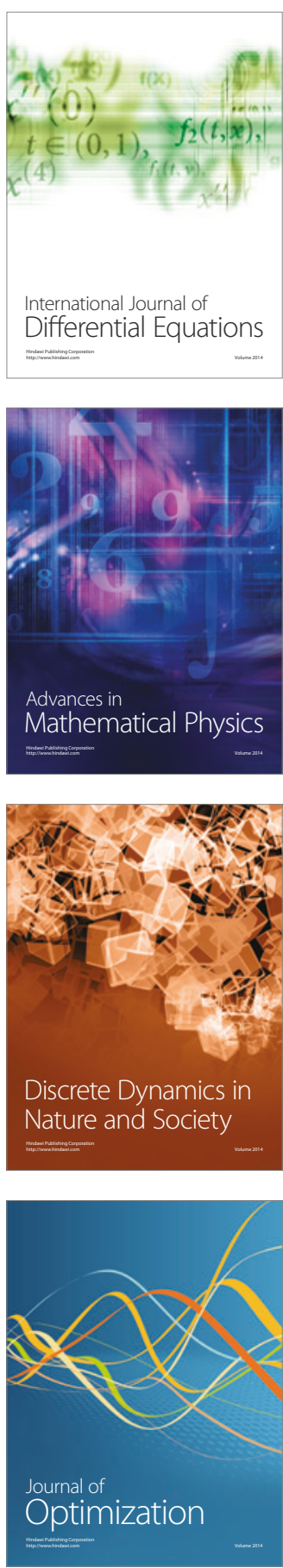